\title{
Determinants of Vaccinal Incompletude in the Context of Implementation of Financing Based on the Performance in Bertoua Health District
}

\author{
Ka'aba Mongo Charles Josias ${ }^{1}$, Bassong Mankollo Olga Yvonne ${ }^{1}$, Assam Assam Jean Paul ${ }^{1,2, *}$ \\ ${ }^{1}$ Department of Public Health, School of Health Science, Catholic University of Central Africa, Yaoundé, Cameroon \\ ${ }^{2}$ Department of Biochemistry, Faculty of Sciences, University of Douala, Douala, Cameroon
}

Email address:

assamjean@yahoo.fr(A. A. J. Paul)

${ }^{*}$ Corresponding author

\section{To cite this article:}

Ka'aba Mongo Charles Josias, Bassong Mankollo Olga Yvonne, Assam Assam Jean Paul. Determinants of Vaccinal Incompletude in the Context of Implementation of Financing Based on the Performance in Bertoua Health District. Central African Journal of Public Health. Vol. 4, No. 4, 2018, pp. 112-118. doi: 10.11648/j.cajph.20180404.13

Received: March 1, 2018; Accepted: March 27, 2018; Published: September 5, 2018

\begin{abstract}
The immunization completion rate of children under 12 months remains low despite the implementation of Financing based on the performance in the Eastern Region of Cameroon. The objective of this study was to identify the determinants of this vaccine incompleteness from 2013 to 2014. A transversal study with descriptive and analytical aim was carried out. A stratified 2-stage sampling was carried out on 309 parents of children and 89 health personnel. A factorial analysis allowed to obtain these children profile according to their situation of complete immunization. Multiple logistic regression was used to control the confounding factors and to establish associations between the variables. The final model was adjusted using chi-square tests and a P-value of $5 \%$ and $10 \%$ was used. Vaccine completive in children under 12 months of age was $44 \%$. The average standard of living $(\mathrm{P}=0.062)$, proximity to the health facility $(\mathrm{P}=0.000)$, the distance of the populations from their health facility $(\mathrm{P}=0.074)$, the recall of the appointment of the vaccination to the parents by the community relay $(\mathrm{P}=0.031)$ Were significantly associated with vaccine incompleteness. $83.9 \%$ of health care providers do not appreciate the distribution of subsidies. No formal contract commits the community relay with the health facilities. District immunization coverage remains low despite the Financing based on the performance. The contractualization of community relays and the practice of good governance would contribute to improving the quality of immunization coverage.
\end{abstract}

Keywords: Vaccine Completeness, Expanded Program of Vaccination, Determinants, Implementation, Financing Based on the Performance

\section{Introduction}

Vaccination is recognized as one of the most effective measures to prevent mortality, morbidity and complications of infectious diseases in children. Through the efforts of governments, international organizations and hundreds of thousands of volunteers, it saves nearly 2.5 million children every year [1]. Two challenges remain to date in the world: reaching every child and making new vaccines available to all children [2].

Aware of the many challenges, and that immunization coverage can not be achieved without further effort, the Global Alliance for Vaccine and Immunization (GAVI) concludes from the experiences of countries such as Rwanda and Haiti that the Financing based on the performance (FBP) is the default approach for cash support to the Strengthening the Health System [3]. This approach has been designed to encourage the improving of immunization outcomes by strengthening the health system in other words, improved immunization outcomes will be rewarded by linking cash support to the performance.

In 2005, the Ministry of Health in Rwanda, with the support of Belgian technical cooperation, launched a performance-based funding strategy [4]. Overall, current health indicators in Rwanda provide evidence of progress over the past 10 years, with a high level of immunization 
coverage in 2005 of more than $90 \%$ of the population. In addition, the infant mortality rate moved from 152 deaths per 1000 live births in 2005 to 103 in 2007 [5].

The FBP approach is taking shape in Cameroon in 2012 by signing a service contract between the Ministry of Public Health and the Cordaid Foundation [6]. Since maternal, child and adolescent health is one of the priority areas of intervention [7, 8]. Despite this new approach, the immunization completion rate for children of less than 12 months remained very low: $47.7 \%$ in the Eastern Region of Cameroon and $41.25 \%$ in the Bertoua [8].

Through this study, we aim to identify the determinants of vaccine non-completion in children under one year of age during the period of FBP implementation in the Bertoua Health Disctrict from 2013 to 2014, improve the coverage of the vaccine by capitalizing the community's potential.

\section{Methodology}

\subsection{Framework of the Study}

This study was carried out among households of the Health District area of Bertoua, one of the 14 Health Disctricts in the Eastern region of Cameroon (pilot region for the implementation of the FBP strategy from June 2012 to December 2014). It covers an area of more than $20000 \mathrm{~km}^{2}$ with a population of 184393 inhabitants.

\subsection{Study Design}

This study is cross-sectional with a descriptive and analytical aim, carried out between December 2015 and November 2016. We administered questionnaires previously pretexted to 309 parents of children under 12 months and to 89 health care providers in health facilities. The sampling for the households in our study was stratified to 2 degrees: the first degree was related to the children actually present during the study period and the second degree concerned the age of the child at the time of the study ( 03 or 04 years). Care providers were selected in a comprehensive manner.

\subsection{Data Collection Tools and Techniques}

Two structured questionnaires were used to conduct our study: The first questionnaire for parents was structured into 06 modules: individual characteristics of the parent, socio-economic characteristics of the parent, geographic characteristics, community participation, mother's health culture, the availability of the child's immunization record and the child's immunization status. The second questionnaire for health professionals was structured into three modules: staff motivation, manipulation of the index tool and motivation of the community relay.

\subsection{Definition of Variables}

The dependent variable is "vaccine incompleteness in children under 12 months" which is a qualitative variable. It was considered as incompletely vaccinated, since all children who had not received all vaccine from the Expanded program of vaccination (EPV) before 12 months of age, including: BCG, OPV0; Penta1, OPV1; Penta2, OVP2; VAM and YFV. Independent variables were the frequency modalities of contextual factors of residence, factors related to health culture, community participation and occupational factors.

\subsection{Data Analysis}

We used the methods of bivariated analysis, multidimensional analysis and logistic regression using SPSS and SPAD-5 for this study. The bivariated analysis allowed us to highlight the extent of the use of vaccination of children according to the different characteristics related to the latter and to measure the degree of community participation. The degree of association was measured using the chi-square statistical test. The analysis of the Multiple Matching enabled us to study the existing associations between the independent variables and the dependent variable. A P $<0.05$ and an adjusted odds ratio with a $95 \%$ confidence interval was considered significant.

\subsection{Ethical Considerations}

Ethical clearance Number 2016/0347/CEIRSH/ESS/MSP was obtained from the Institutional Ethics Committee of the Catholic University of Central Africa (CUCA). An authorization Number 348/L/MINSANTE/SG/DRSPE/BFP/BCASS from the Eastern Regional Public Health Delegation in Cameroon was obtained prior to the start of data collection. Informed consent was required from each participant prior to inclusion in the study. All subjects agreeing to participate in the study had to confirm their consent either by signature or tacit agreement.

\section{Results}

\subsection{Individual Characteristics of Children Under 12 Months of Age Under the FBP}

Of the 309 children studied, 57.3\% were male and 43.4\% female. Very few of these children $(8.1 \%)$ did not have a vaccination record. More than half of these children (56\%) had not completed routine immunization of the EPV program. Health culture, prenatal clinic services are not particularly needed. Only $11.3 \%$ of mothers of children were able to perform 3 prenatal consultations. Prenatal consultation is not much sought, $39.5 \%$ do not do it. In addition, the total number of deliveries outside a health facility was $42.1 \%$ as illustrated in Table 1 .

Table 1. Distribution (\%) of children's own characteristics.

\begin{tabular}{lll}
\hline Characteristics & Terms & Percentage (\%) \\
\hline \multirow{2}{*}{ Sex } & Male & 57.3 \\
Availability of the book & Female & 42.4 \\
vaccination & Yes & 91.9 \\
\multirow{2}{*}{ status immunization } & No & 8.1 \\
& Full & 44 \\
Number of prenatal & Non full & 56 \\
consultations for the child & 3PNC & 11.3 \\
& 2PNC & 12 \\
\hline
\end{tabular}




\begin{tabular}{lll}
\hline Characteristics & Terms & Percentage (\%) \\
\hline & No & 39.5 \\
& Home & 21.7 \\
Place of birth & midwife traditional & 20.4 \\
& Health Center & 57.9 \\
\hline
\end{tabular}

\subsection{Characteristics of the Living Environment of the Children Studied}

Table 2 shows that the majority of the parents surveyed (74.4\%) were male. Two main religions emerged from the spiritual practice of these parents: the Christian religion $71.2 \%$ and the Muslim religion 26.5\%. Most of the parents surveyed $(53.1 \%)$ were socialized in medium-sized towns, compared with $17.8 \%$ in the villages. The standard of living of the households visited as a whole is low with $71.2 \%$ living below the poverty line. Sixty-nine percent $(69.9 \%)$ of the parents surveyed live in urban areas and $30.1 \%$ in rural areas. With regard to the distance between health facilities and parents' homes, $42.2 \%$ are far away.

Table 2. Distribution (\%) of characteristics of children's living environment.

\begin{tabular}{lll}
\hline Characteristics & Terms & Percentage (\%) \\
\hline \multirow{2}{*}{ Sex parent } & male & 74.4 \\
& female & 25.6 \\
Parents religion & Muslim & 26.5 \\
& Christian & 71.2 \\
& without religion & 2.3 \\
Place of socialization & city average & 53.1 \\
& small town & 29.1 \\
& village & 17.8 \\
Educational level & without level & 14.9 \\
& primary & 32.4 \\
& secondary & 40.1 \\
& higher & 12.6 \\
Socio-professional category & cultivator & 55 \\
& public sector & 7.1 \\
& private sector & 2.9 \\
& informal sector & 35 \\
Standard of living & low & 71.2 \\
& average & 21.7 \\
Geographical accessibility & high & 7.1 \\
& urban & 30.1 \\
& Rural & 69.9 \\
& close & 25.6 \\
& & 23.6 \\
& very remote & 50.8 \\
\hline
\end{tabular}

\subsection{Knowledge and Level of Access to Community Relay} Information

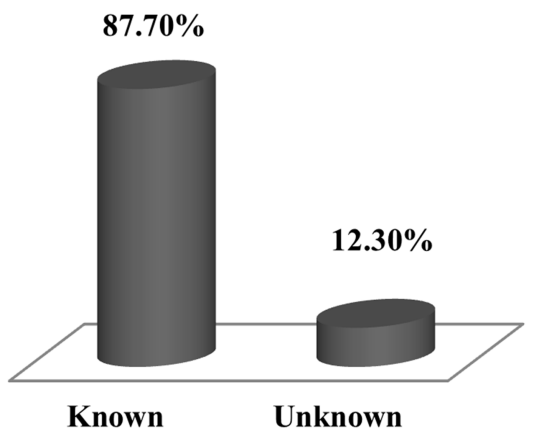

Figure 1. Distribution of parents according to the knowledge of the CR.

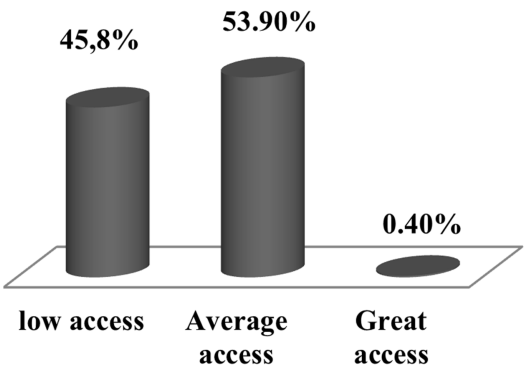

Figure 2. Distribution of parents according to access to information of the CR.

Figures 1 and 2 show that community relay (CR) agents are well known to most parents surveyed, $87.7 \%$ of parents say they know the community relay in the region. Only $0.4 \%$ of parents have very high access to information from community relays compared with $53.9 \%$ who say they have little access to this information.

\subsection{Appointment Recall Strategy and Opportunity to Meet with Community Relay}

Figures 3 and 4 respectively illustrate the most used strategy by $\mathrm{CR}$ to recall the appointment of vaccination programs which is the home visit $(88 \%)$ and the occasions when parents are in contact with the $\mathrm{CR}$ are national or regional vaccination campaigns $(85.6 \%) .11 .4 \%$ of parents benefit from the reminder of vaccination appointments by the CR.

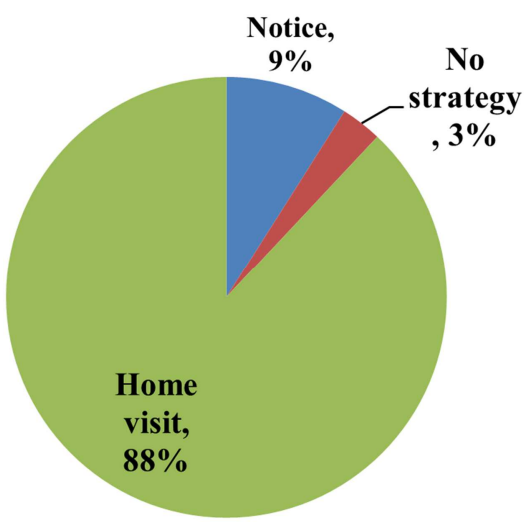

Figure 3. Distribution of the type of strategy for recall of appointments by the $C R$.

\section{$85.60 \%$}

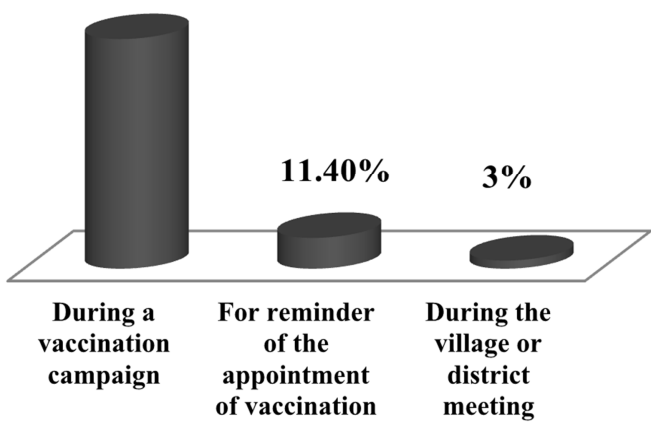

Figure 4. Distribution of opportunities for meeting with CR. 


\subsection{Identification of the Characteristics of Children Under 12 Months in Direct Links with the Situation of Complete Immunization}

Educational attainment, socio-occupational category, standard of living, place of residence, geographic accessibility are significantly associated with completeness of the vaccine at the $1 \%$ threshold as shown in Table 3; The type of strategy used by the community relay and the knowledge of $\mathrm{CR}$ are significantly associated with immunization completeness at the $1 \%$ threshold $(\mathrm{P}=0.000)$;
The opportunity to meet the $\mathrm{CR}$ by households is related to the completeness of the vaccine at the significance level of $5 \%(\mathrm{P}=0.000)$. The number of NPCs, the place of delivery are significantly associated with immunization complete at the $1 \%$ threshold $\mathrm{P}=0.000$. The availability of the vaccination record is significantly related to the completeness of the vaccine at the $5 \%$ threshold $(\mathrm{P}=0.012)$; The sex of the child is the last characteristic significantly associated with vaccine complete at the $5 \%$ threshold $(\mathrm{P}=0.04)$.

Table 3. Degree of linkage between the characteristics of the children and the situation of the completeness of the vaccination.

\begin{tabular}{|c|c|c|c|}
\hline Characteristics & Value of KHI-two & P-value & Decision \\
\hline Parents religion & 1.627 & 0.443 & unrelated \\
\hline Place of socialization & 1.611 & 0.65 & unrelated \\
\hline Education $* * *$ & 19.102 & 0.000 & related \\
\hline Socio-professional category $* * *$ & 63.098 & 0.000 & related \\
\hline Standard of living $* * *$ & 104.31 & 0.000 & related \\
\hline Place of residence $* * *$ & 169.237 & 0.000 & related \\
\hline geographical accessibility $* * *$ & 148.529 & 0.000 & related \\
\hline Knowledge of CR $* * *$ & 24.814 & 0.000 & related \\
\hline Opportunity to meet the CR ** & 37.619 & 0.000 & related \\
\hline Type strategy of CR *** & 29.517 & 0.000 & related \\
\hline Level of access to CR information & 0.833 & 0.659 & unrelated \\
\hline Number of PNC $* * *$ & 74.883 & 0.000 & related \\
\hline Place of birth $* * *$ & 23,198 & 0,000 & related \\
\hline Child sex ** & 6.349 & 0.04 & related \\
\hline Availability of the book vaccination $* *$ & 6.365 & 0.012 & related \\
\hline Age group & 0.145 & 09.86 & unrelated \\
\hline
\end{tabular}

$(*)$ : significant to $10 \% ;(* *)$ significant to $5 \%$; $(* * *)$ significant to $1 \%$.

\subsection{Factorial Map of Children Under 12 Years According to the Situation of Completed Vaccination}

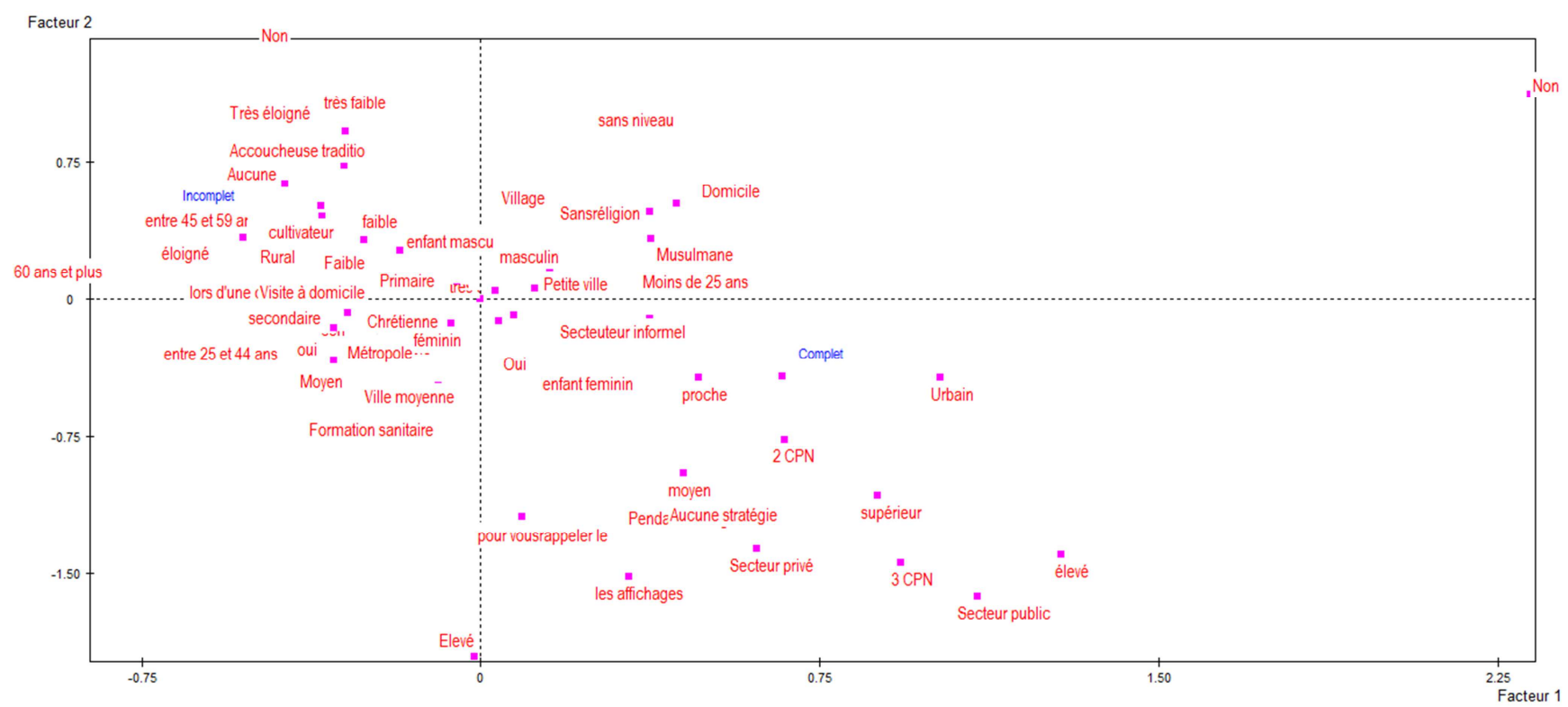

Figure 5. Children's Factor 1-2 Plans by Immunity Status.

Factor 1-2 (Figure 5) shows the existence of two groups of children with different profiles. The group of children who had an incomplete vaccination status and those with full vaccination status. The first group with incomplete immunization consists mainly of male children living in rural areas. The parents of these children who are often of advanced age also very often use the services of a traditional birth attendant, which explains why they did not have to make any prenatal visits. In terms of accessibility to the nearest health facility, they often live very far from health facilities. The second group of children with full immunization status consists of living children in close 
proximity to health facilities. Their parents have a generally high standard of living. Moms who are very often from a young age have had to do at least 02 prenatal consultations. As for access to communal communications, parents of fully immunized children are regularly visited by the CR

\subsection{Evaluation of the Risks of Non-Complemencies of Vaccination of the Less than 12 Months According to Their Individual Characteristics}

As shown in Table 4, the logistic regression carried out reveals that geographical accessibility increases the risk of complete immunization. Children with dwellings close to health facilities have 238 times the chance of completing their vaccines with a $\mathrm{P}$. value less than 0.001 at the $1 \%$ threshold and 95\% CI $=$ [27.7-2052.6]. Children far away from health facilities are 7 times more likely to complete their vaccine, with $\mathrm{O} . \mathrm{R}: 7.733 \mathrm{P}=0.039$ and a $95 \% \mathrm{CI}=$ [1.138-17.032]. This last result can be explained by the action of the CRs whose recall of the appointments of vaccination to the parents increases 23 times more the risk of completing his vaccines, with a P. value of 0.031 and a $95 \%$ $\mathrm{CI}=1.339$ to 428.6$]$. In addition, children born outside of Health facility are 04 times more likely to complete their vaccines, with a $\mathrm{P}$. value 0.032 and a $95 \% \mathrm{CI}=[1.138$ 17.032]. Children whose parents have an average standard of living are 3 times more likely to complete their vaccinations with a $\mathrm{P}$. value of 0.062 and a $95 \% \mathrm{CI}=[4.12-14.03]$.

Table 4. Odds-Ratio values according to child characteristics.

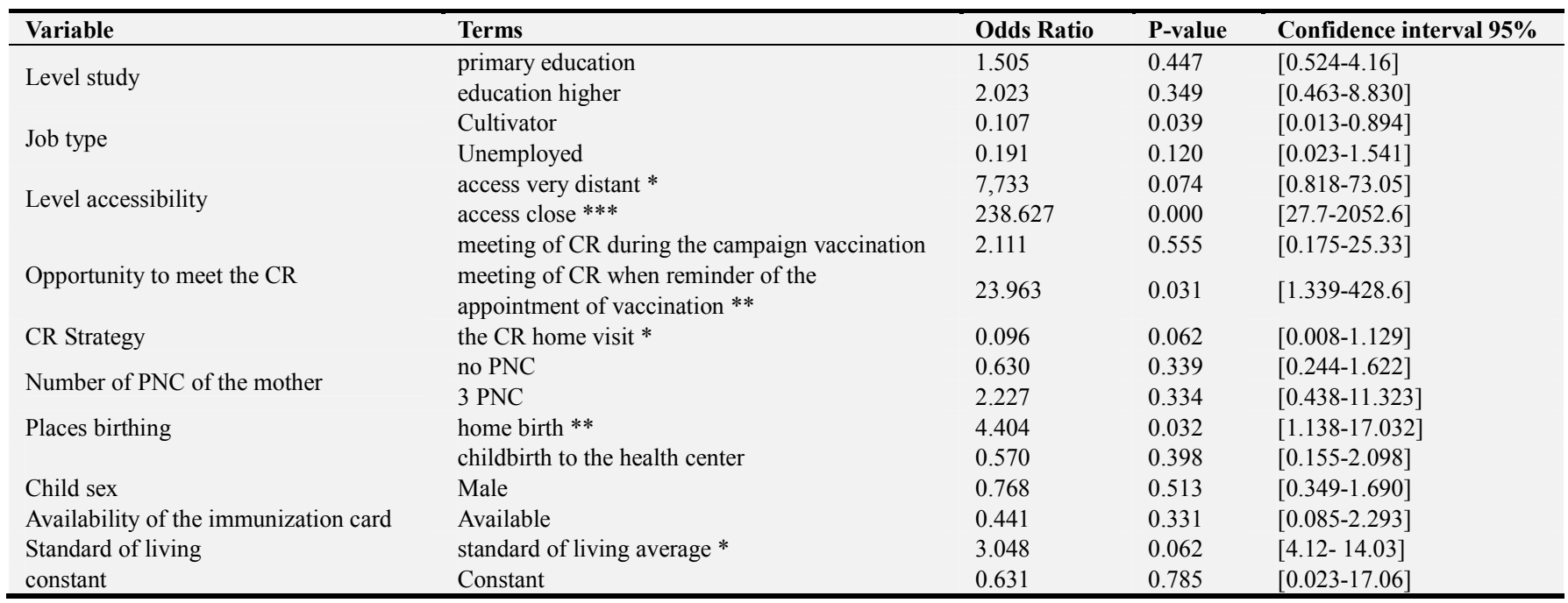

\subsection{Status of Governance and Community Participation in Low-Performance Vaccine Completion Health Facilities for Children Under 12 Months of Age}

Only $18.1 \%$ of claimants believe that the distribution of subsidies is good, with the majority disagreeing. Opinions are opposed on the value of the indicator for purchase by the performance buying agency (PBA) as shown in Table 4. $49.4 \%$ think it is poorly paid, and $50.6 \%$ think otherwise. Only $16.9 \%$ of staff believes that the payment deadlines for subsidies are respected. The index tool does not seem to fit into the practice of allocating subsidies since only $6 \%$ of staff have knowledge of the index tool. $12 \%$ of the staff still use the services of the CRs, $74.7 \%$ admit to having recourse to the services of the RCs from time to time. There is no formal contract between the $\mathrm{CR}$ and health facilities according to $72.3 \%$ of health personnel. No CR has planning in the business plan or budget of the health facility, $100 \%$ of the health personnel recognize it. $94 \%$ of staff believe that $\mathrm{CR}$ is essential for immunization activities.

\section{Discussion}

Our study revealed that $44 \%$ of children under 12 months of age were fully vaccinated in the Bertoua, compared with $56 \%$ of children who had not completed their EPV vaccine. This result confirms that of the PBA evaluation report which found an average vaccine completeness rate for the years 2013 and 2014 at $41.25 \%$. These results as a whole are slightly lower than those rendered by the EDS-MICS 2011 [9] which gives the vaccine completeness rate of less than 12 months to $48 \%$. They are also low compared to those obtained in 2012 by Baonga Ba Pouth et al. in 2014 [10] in the health District of Djoungolo (Yaoundé) in Cameroon with $64.3 \%$ of children completely vaccinated. This performance may justify the circulation of wild polio virus in the Eastern Region in 2013 and 2014 and sporadic epidemics of measles during these same years [11]. Implementation of FBP during this period should have contributed to the improvement of immunization completeness. A study by louis Rusa et al. in 2007 [5] on the quality of services in health facilities in Rwanda in the context of FBP showed an increase in immunization coverage to $90 \%$ of children before their first birthday. This vaccination performance of the Bertoua health district compared to that of Rwanda is proof that FBP has not contributed sufficiently to the improvement of the complete vaccination coverage in children under 12 months.

Several determinants have been identified in this study to 
explain the incomplete immunization in children under 12 months of age. Those who received our attention were significantly associated with the completeness of the vaccine in these children in the context of FBP. These are due to the contextual factors of living standards and geographical accessibility; Factors related to community participation such as meeting with parents to remind them of the immunization appointment and occupational factors that were developed through the use of frequencies related to the analysis of the questionnaire administered to health professionals.

Our results showed that the standard of living was significantly associated with immunization completeness at the $1 \%$ threshold. With 3 times more risk for children under 12 months of households with average incomes to complete EPV vaccines [OR: $3.048 ; \mathrm{P}=0.062$ ]. These findings are in line with those of Guthmann et al., in 2013 [12] in a report by the health surveillance institute on the socioeconomic determinants of BCG and pneumococcal vaccinations among children in the Paris region. Socio-economic factors investigated, household income or standard of living was the only factor significantly associated with immunization coverage [13]. In addition, in its 2011 report, the EDS-MICS recognizes that the average household's standard of living positively influences the use of health care for the child (EDS-MICS 2011) [7]. The FBP will not have reached the poorest households in terms of vaccination for children under 12 months of age.

Our results showed that $69.9 \%$ of the parents surveyed live in urban areas and $30.1 \%$ in rural areas. They also showed a correlation between immunization completeness and the context in which the parents live. Gilmore in 2010 found in his study on the strategies and determinants of immunization in Burkina Faso 1993 - 2003 that complete vaccination coverage increased in rural areas, from $25.9 \%$ to $41.2 \%$ between 1998 And 2003 [14]. As the health areas of the Bertoua health district are largely in rural areas, the Reach each District (RED) approach needs to be revitalized. The FBP presents itself as an opportunity to overcome this inequality.

Our results showed a significant association with educational attainment of $1 \%$. Indeed we obtained $14.9 \%$ without level and $52.7 \%$ of the secondary level and more. Baonga Ba Pouth et al. (2014) [10] in their work on the factors associated with vaccine incompleteness in the health district of Djoungolo showed that $64.8 \%$ of heads of households had a high school education and more. While $7.1 \%$ had no education. The importance of the role of parent education seems undeniable in the completeness of vaccination in children under 12 months of age. However, is the illiteracy of the parents always responsible for the incompleteness of the vaccination?

Our study shows that geographic accessibility has a significant association with vaccine completeness. The proximity of the health facility gives 238 times more chance for children under 12 months of the Bertoua health district to complete the vaccines. Our results are consistent with those of Jamil, Bhuiya who find that the probability of a child being fully vaccinated is related to the distance from the nearest health facility [15]. Remoteness is a serious handicap to the complete immunization of children under 12 months of age. Our results also indicated that populations far away from their health facilities are 7 times more likely to complete their vaccines. It can therefore be assumed that some very remote populations benefit from a regular descent of a vaccination team. However, geographic accessibility remains dependent on the environment. Indeed, being $5 \mathrm{~km}$ from a health service certainly does not have the same realities in all environments, with regard to the efforts to provide (mountain, streams, road conditions).

Our study shows a significant association at the $1 \%$ threshold between the chance of CR meeting with vaccine completeness. Children of parents who have benefited from the recall of the vaccination appointment from the CR have 23 times more chance of finishing their vaccine. In addition, our study showed that $86.7 \%$ of health professionals acknowledge the use of the CR service. Despite the fact that we did not have a study on FBP correlated with the involvement of the community relays, we nevertheless note the importance of the community relays within the framework of the vaccination. Indeed, in a study on the evaluation of the contribution of community relays to improve immunization coverage in the Banamba district of Mali (2001-2005), Cissé, in 2006 [16] showed that the contribution of community relays has been positive both on the incidence of immunization coverage and on the improvement of mothers' knowledge about vaccination. This result raises a reflection on the role and the contribution of the CRs in the context of implementation of the FBP, to improve the immunization coverage in children of less than 12 months in the Bertoua health district. The role of community relays in the health system is very important and essential for the improvement of health indicators

Our study shows that the majority of health workers (more than $70 \%$ ) do not appreciate the distribution of subsidies that do not reflect the reality of the revenues and that they generally do in an arbitrary way. Most of them, 96\%, say they do not know what the index tool is, which is an instrument that allows the equitable distribution of subsidies according to the previously calculated calculation indices. This poor appreciation of the staff as a whole is evidence of the problems of poor governance in Cameroon's public services [17].

\section{Conclusion}

The completeness of vaccination in children under 12 months remains very low despite the strategy of the FBP set up in the Bertoua health district from 2012 to 2014. The determinants responsible for this incomplete immunization in these children are: the standard of living of the household, Geographical accessibility to Health facility, poor governance in Health facility, non-involvement of community outreach agents in the FBP process, and underutilization of maternal health services by mothers. 
Improved coverage Vaccination requires the capitalization of the potential of the community relays through their contracting and the practice of good governance.

\section{Conflicts of Interest}

The authors declare that they have no competing interests.

\section{Author Contributions}

Design and Development of the Protocol

KA'ABA MONGO Charles Josias; Olga Yvonne BASSONG MANKOLLO.

Data Collection

KA'ABA MONGO Charles Josias; Olga Yvonne BASSONG MANKOLLO; ASSAM ASSAM Jean Paul.

Data Analysis

KA'ABA MONGO Charles Josias; Olga Yvonne BASSONG MANKOLLO.

Interpretation of Results

KA'ABA MONGO Charles Josias; Olga Yvonne BASSONG MANKOLLO. Writing of the manuscript: KA'ABA MONGO Charles Josias; Olga Yvonne BASSONG MANKOLLO; ASSAM ASSAM Jean Paul.

All authors have read and approved the final version of the manuscript.

\section{Acknowledgements}

We thank the Regional Public Health Delegate for the authorization of this study, the Chief of Health Service of the District of Bertoua and the Administrator of the Regional Fund for facilitating access to his services.

\section{References}

[1] UNICEF-OMS (2010). Les soins de santé primaires, UNICEF-OMS Genève.

[2] OMS (2014). Rapport sur la santé dans le monde: Le financementdes systèmes de santé: le chemin vers une couverture universelle, Genève.

[3] Meissen, B., Soucat, A., Sekabaraga, C. (2011). PerformanceBased Financing: Just a DonorFad or a Catalyst Towards Comprehensive Health-Care Reform? Bulletin de l'Organisationmondiale de la Santé 89, 53-56.

[4] Meessen, B., Musango, L., Kashala, J.-P., Lemlin, J. (2006). Reviewing Institutions of Rural Health Centres: The Performance Initiative in Butare, Rwanda », Tropical Medicineand International Health 11, 13-17.
[5] Rusa, L., Ngirabega, J.-D., Janssen, W., Van Bastelaere, Porignon, D. and Vandenbulcke, W. (2009). Performancebased financing for better quality of services in Rwandan health centres: 3-year experience. Tropical Medicine and International Health.

[6] Ministère de la santé publique: STRATEGIE SECTORIELLE DE SANTE 2001 - 2015 au Cameroun.

[7] Mumssen, Y., L. Johannes, L., Kumar, G. (2010). OutputBased Aid: Lessons Learned and Best Practices, Washington, DC: Banquemondiale.

[8] Institut National de la Statistique (INS) et ICF. International (2012) Enquête Démographique et de Santé et à Indicateurs Multiples du Cameroun 2011. Calverton, Maryland, USA: INS et ICF International.

[9] Gilmore, M. E. (2013). Effectiveness of community health workers delivering preventive interventions for maternal and child health in low- and middle-income countries: a systematic review. BMC Public Health, p. 13.

[10] Baonga Ba Pouth, S. F., Kazambu, D., DieulaDelissaint, Kobela, M. (2014) Couverture vaccinale et facteurs associés à la non complétude vaccinale des enfants de 12 à 23 mois du district de santé de Djoungolo-Cameroun en 2012. Pan AficanMedical Journal.

[11] Bonono, R. C., Ongolo-Zogo P. (2012). Optimiser l'utilisation de la consultation prénatale au Cameroun. Centre pour le Développement des Bonnes Pratiques en Santé - Hôpital Central. Yaoundé, Cameroun.

[12] Guthmann, J-P., Chauvin, P., Le Strat, Y., Soler, M., Fonteneau, L., Lévy-Bruhl, D. (2013). Déterminants socioéconomiques des vaccinations BCG et pneumocoque chez les enfants de la région parisienne, Résultats de l'enquête VACSIRS, 2010. Saint-Maurice: Institut de veille sanitaire. http://www.invs.sante.fr.

[13] Fournier, P., Potvin, L. (1995). Participation communautaire et programmes de santé: les fondements du dogme. Sciences sociales et santé. Volume 13, n². 39-59.

[14] Document de recherche de politique 6364, Banque mondiale, Washington, DC (2013).

[15] Brahima, A. D. (2013). Déterminants socio-culturels de l'accès et l'utilisation des services de santé maternelle et néonatale au Mali: Cas de la Commune I du district de Bamako. Programme AFD-Sahel, rapport pays MALI AFRIKCONSULTING.

[16] Cissé, B. (2006). Evaluation de l'apport des relais communautaires sur l'amélioration de la couverture vaccinale dans le district de Banamba au Mali (2001 - 2005).

[17] Mba, R. M., Ongolo-Zogo, P. (2011). Améliorer la gouvernance pour la viabilisation du district de santé au Cameroun. CDBPS. 